\title{
Perineural invasion in endometriotic lesions contributes to endometriosis-associated pain
}

This article was published in the following Dove Press journal: Journal of Pain Research

\author{
Yanchun Liang' \\ Duo Liu' \\ Fan Yang' \\ Wenwei Pan' \\ Feitianzhi Zeng' \\ Jinjie $W u^{2}$ \\ Hongyu $\mathrm{Xie}^{2}$ \\ Jiaying $\mathrm{Li}^{2}$ \\ Shuzhong Yao' \\ 'Department of Obstetrics and \\ Gynecology, The First Affiliated \\ Hospital, Sun Yat-sen University, \\ Guangzhou 510080, China; \\ ${ }^{2}$ Zhongshan School of Medicine, \\ Sun Yat-sen University, Guangzhou \\ 510080 , China
}

Correspondence: Shuzhong Yao Department of Obstetrics and Gynecology, The First Affiliated Hospital, Sun Yat-sen University, No. 58, The 2nd Zhongshan Road, Yuexiu District, Guangzhou 510080, Guangdong Province, China

Tel +86 I 3602834 I 27

Email yszlfy@।63.com
Purpose: Recent studies have shown that abnormal distribution of pelvic nerves contributes to endometriosis-associated pain. However, the relationship between neurogenesis and pain severity in endometriosis still remains uncertain, which makes it an enigma for both gynecologists as well as neuropathologists. In this study, we tried to explore a special phenomenon, perineural invasion (PNI), in deep infiltrating endometriosis (DIE) and investigated the correlation between PNI- and DIE-associated pain.

Patients and methods: The study was conducted in the Department of Obstetrics and Gynecology of the First Affiliated Hospital of Sun Yat-sen University from June 2012 to January 2015. In total, 64 patients with DIE were enrolled. They received laparoscopically surgical resection of endometriotic lesions. The Kruskal-Wallis and Mann-Whitney tests were used for comparisons of enumeration data. Spearman rank correlation was used for linear analysis. Results: Immunohistochemical analysis demonstrated that PNI was commonly found in DIE lesions. Patients were divided into PNI (+) group and PNI (-) group. The visual analog scale scores of dysmenorrhea, dyspareunia, and chronic pelvic pain were higher in PNI (+) group than in PNI (-) group. Also, we found significantly increased density of newly formed nerve fibers as well as microvessels in lesions of PNI (+) group. Further, double immunofluorescence showed a closely spatial nerve-vessel network in the endometriotic lesion of PNI (+) group. More importantly, correlation analysis revealed positive relation between the density of newly formed nerve fibers in the lesion and the density of microvessels in lesions of PNI (+) group. Conclusion: This study suggests that PNI in endometriotic lesions plays an important role in endometriosis-associated pain, mainly through a mechanism named "neuroangiogenesis".

Keywords: perineural invasion, deep infiltrating endometriosis, pain, neuroangiogenesis

\section{Introduction}

Endometriosis is a well-known gynecologic disease affecting $10 \%-15 \%$ of women of reproductive age worldwide. It remains an enigmatic disorder due to its multiple symptoms and complicated pathogenic mechanisms. Dysmenorrhea, dyspareunia, and chronic pelvic pain are the most common pain symptoms attributed to endometriosis. However, the mechanism underlying EAP remains unknown.

Several lines of clinicopathological evidences suggest that abnormal distribution of pelvic nerve fibers plays an important role in the generation of EAP. ${ }^{1-3} \mathrm{NFD}$ is significantly increased in peritoneum and endometriotic lesion in patients with peritoneal endometriosis compared with normal peritoneum. ${ }^{4}$ This interesting phenomenon is mainly due to the abnormal neurogenesis within the endometriotic lesion resulting from 
different mechanisms. Berkley et al reported that ectopic endometriotic lesion could develop its own autonomic and sensory innervation both in rats and women. ${ }^{5,6}$ Mechsner et al found stronger expression of GAP-43 (a marker for neural outgrowth and regeneration) in endometriosis-associated nerve fibers than nerves distant from endometriotic lesions. ${ }^{7}$ Furthermore, they discovered a close localization between endometriosis-associated nerve fibers and immature blood vessels within the stroma, suggesting that the nerve-vessel interaction (neuroangiogenesis) ${ }^{8}$ may lead to the generation of new nerve fibers. Neurotrophic factors such as NGF and NT-3 produced and released by ectopic endometrial cells are increased in endometriotic lesions, also providing a nerve growth-promoting environment. ${ }^{9,10}$

However, limited studies demonstrate a direct relationship between the presence of certain nerve fibers and the severity of pain symptoms in endometriosis. Mechsner et al found an increase of peritoneal endometriosis-associated nerve fibers in patients with higher VAS score. ${ }^{11}$ Our previous study also revealed parallels between NFD and pain severity in peritoneal endometriosis as well as DIE. ${ }^{12} \mathrm{~A}$ better understanding of the etiology of EAP is of great clinical significance to improve the treatment of women who suffer from endometriosis. As a consequence, more clinicopathological investigations are needed to further reveal the relationship between nerve fibers and endometriotic pain symptoms.

This study focused on exploring potential factors contributing to the generation of pain in DIE from a special view of estimating the interaction of ectopic endometrial cells and nerve fibers. We examined the percentage of PNI in DIE and tried to explore the correlation between PNI and the severity of pain symptoms. Additional experiments were performed to investigate the density of newly formed nerve fibers as well as microvessels. Our data provide evidence that PNI may play an important role in pain generation in endometriosis.

\section{Patients and methods}

\section{Patients}

The study was conducted in the Department of Obstetrics and Gynecology of the First Affiliated Hospital of Sun Yatsen University from June 2012 to January 2015. In total, 64 patients with DIE (mean age 32.5 years, range 20-42 years) were enrolled and received laparoscopically surgical resection of endometriotic lesions. Tissues samples of endometriotic nodules infiltrating the uterosacral ligament and/or rectovaginal septum were collected. Subject inclusion criteria included the following: 1) all patients were premenopausal with a regular menstrual cycle; 2 ) postoperative pathologi- cal evidence confirmed endometriosis; 3) all patients presented with pain symptoms (dysmenorrhea, dyspareunia, and chronic pelvic pain) for more than 6 months. Patients were surgically treated because of their pain symptoms, not because of infertility. Subject exclusion criteria included the following: 1) patients with malignant disease; 2) patients with chronic inflammatory diseases; 3 ) patients with histological confirmed adenomyosis or patients with clinical highly suspected adenomyosis (either endovaginal ultrasonographical or magnetic resonance imaging suspicion); 4) endometriosis involving the bowel wall; 5) patients who had received hormonal treatment for at least 3 months prior to surgery.

The research protocol was approved by the Research Ethics Committee of the First Affiliated Hospital of Sun Yatsen University. All patients provided their written informed consent for this study.

\section{Pain scale assessment}

This self-reporting of pain is considered as the "gold standard" of pain measurement. ${ }^{13}$ As a result, the severity of dysmenorrhea, dyspareunia, and chronic pelvic pain before surgery was assessed by the VAS. We evaluated VAS scores of dysmenorrhea, dyspareunia, and chronic pelvic pain for the recent 6 months. The value of VAS was collected by the investigator who was responsible for preoperative data collection.

\section{Immunohistochemistry and dual immunofluorescence}

Each sample of endometriotic lesion was sectioned at intervals of $0.5-0.7 \mathrm{~cm}$, and all of the sections were routinely processed and embedded in paraffin for histological examination. Then, the sections, stained with hematoxylin and eosin, were examined pathologically (two blinded pathologists) to confirm the diagnosis of endometriosis (the presence of ectopic endometrial epithelial cells and/or the stromal cells). Serial sections ( $4 \mathrm{~mm}$ ) of each focus were processed.

Immunohistochemistry was performed as previously described. ${ }^{12}$ Antibody against a pan neuronal marker, PGP 9.5 (Anti-PGP 9.5, monoclonal mouse, dilution 1:500; Abcam, Cambridge, UK), was applied to identify the intact nerve fibers. Antibody against GAP-43 (Anti-GAP-43, monoclonal mouse, dilution 1:100; Abcam, UK) was used to identify newly formed nerve fibers. Negative control sections were processed by omitting the primary antibody.

PNI was defined as the presence of ectopic endometrial epithelial cells and/or the stromal cells tracking along or around a nerve within the perineural space ${ }^{14,15}$ (Figure 1A, white arrow). Newly formed NFD (number of nerve fibers 
per square millimeter) was recorded by calculating the average number of GAP-43 positively stained nerve fibers, using a grid of $1 \mathrm{~mm}^{2}$, in five nonoverlapping randomly selected high-power fields (magnification 200×). ${ }^{2}$

Dual immunofluorescence was performed to demonstrate the spatial relationship between nerve fibers and microvessels. The method and process of dual immunofluorescence was described in detail in our previous study. ${ }^{12}$ Antibody against CD31 (polyclonal rabbit, dilution 1:2,000; Abcam, UK) was used to identify endothelial cells in neoangiogenesis. ${ }^{16}$ Antibodies for identification of nerve fibers (intact nerve fibers or newly formed nerve fibers) were the same as those used in immunohistochemistry. Differently, DyLight 488 AffiniPure Goat Anti-Mouse IgG and DyLight 594 AffiniPure Goat Anti- Rabbit IgG (EarthOx, LLC, San Francisco, CA, USA) were used as secondary antibodies. Nerve fibers were found green-stained under the observation of a fluorescence microscope. Similarly, red-stained endothelial cells were identified and the number of CD31-stained vessels in the lesion was counted under $200 \times$ magnification, and the number of microvessels per square millimeter lesion (microvessel density [MVD]) was

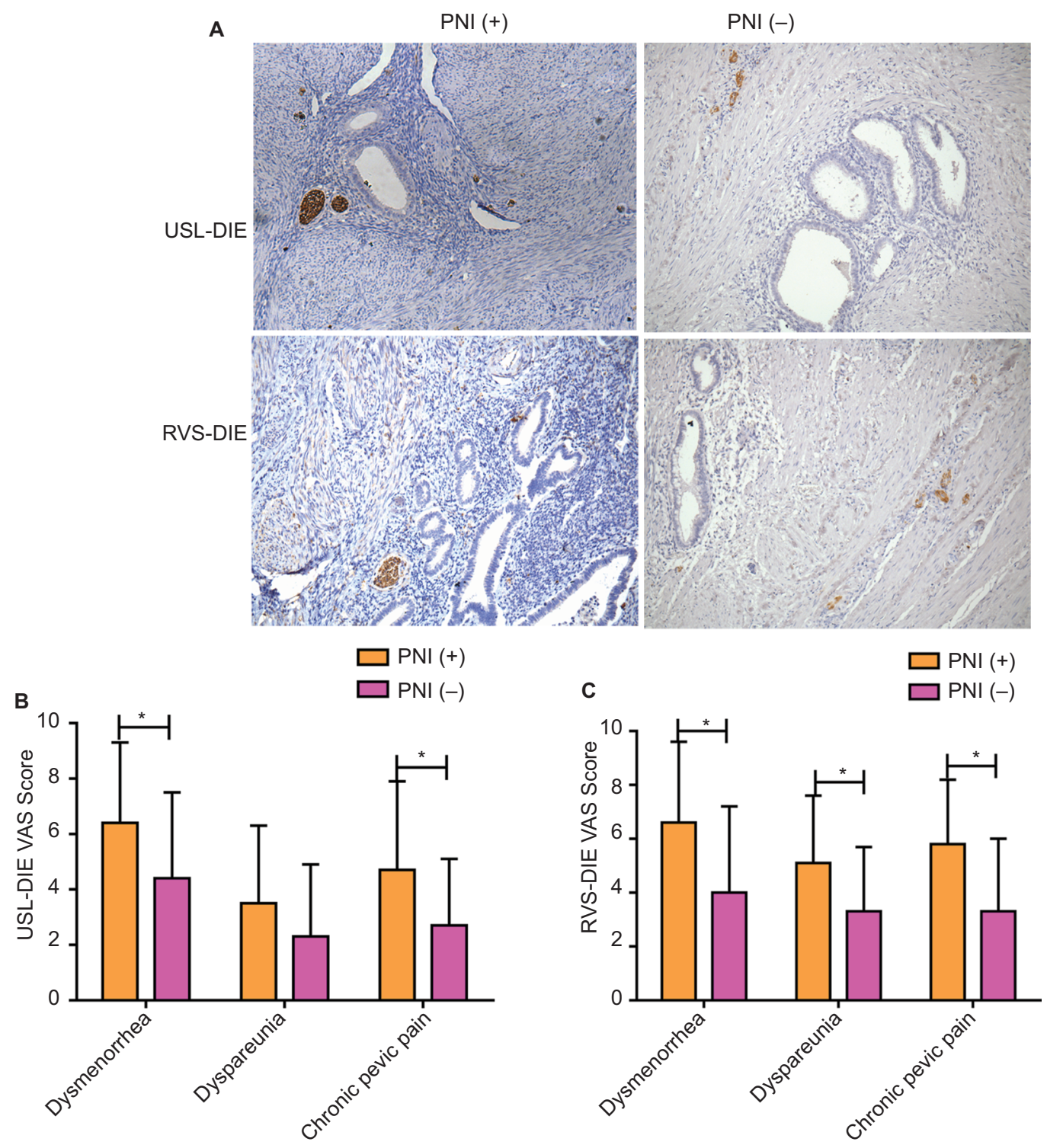

Figure I PNI in deep infiltrating endometriosis and its relationship with EAP.

Notes: (A) Immunohistochemistry (200x magnification) was performed in lesions of USL-DIE and RVS-DIE. Nerve bundles surrounded by ectopic endometrial epithelial cells and/or the stromal cells are shown by white arrows. Patients were divided into two groups: PNI (+) group and PNI (-) group. Overall, 65.5\% (38/58) of USL-DIE were PNI (+) and 70.0\% (28/40) of RVS-DIE were PNI (+). (B and C) VAS scores of dysmenorrhea, dyspareunia, and chronic pelvic pain were collected in all of the patients before the operation. VAS scores were compared between PNI (+) group and PNI (-) group. VAS scores of dysmenorrhea, dyspareunia, and chronic pelvic pain were all higher in $\mathrm{PNI}(+)$ group than in PNI (-) group (USL-DIE: $P=0.020, P=0.01 \mathrm{I}, P=0.054$; RVS-DIE: $P=0.03 \mathrm{I}, P=0.024, P=0.044$ ). $* P<0.05$.

Abbreviations: PNI, perineural invasion; EAP, endometriosis-associated pain; USL-DIE, endometriosis infiltrating the uterosacral ligament; RVS-DIE, endometriosis involving the rectovaginal septum; VAS, visual analog scale. 
calculated. ${ }^{17}$ At least five fields were examined for each slide. Each slide was examined by two blinded observers, and the average of the counts was taken.

\section{Statistical analysis}

Statistical analysis was performed using GraphPad Prism software version 6 (PRISM, La Jolla, CA, USA). Descriptive statistics (mean and SDs) were calculated for each compared group. The Kruskal-Wallis and Mann-Whitney tests were used for comparisons of NFDs and MVDs because they were not normally distributed. Spearman rank correlation was used for linear analysis between NFDs of newly formed nerve fibers and MVDs in endometriotic lesions of PNI (+) DIE patients. $P<0.05$ was considered statistically significant.

\section{Results}

\section{$\mathrm{PNI}$ in DIE}

After surgery, 58 of 64 cases were histologically confirmed USL-DIE, and 40 of 64 cases endometriosis involving the rectovaginal septum (RVS-DIE). In total, 34 cases had both USL-DIE and RVS-DIE.

According to the definition described above, 65.5\% $(38 / 58)$ of USL-DIE were PNI (+); and this ratio was up to $70.0 \%(28 / 40)$ in patients with RVS-DIE (Figure 1A).

\section{Relationship between $\mathrm{PNI}$ and pain symptoms in DIE}

Patients with USL-DIE were divided into two groups: PNI (+) group and PNI (-) group. The VAS scores of dysmenorrhea, dyspareunia, and chronic pelvic pain of all patients were collected before surgery. VAS scores of dysmenorrhea and chronic pelvic pain were significantly higher in PNI (+) patients than in PNI $(-)$ patients $(P=0.020$ and $P=0.011$, respectively). The VAS score of dyspareunia of PNI (+) group was higher than that of PNI (-) group, but the difference was not statistically significant ( $P=0.054$; Table $\mathrm{S} 1$ and Figure 1B). In patients with RVS-DIE, VAS scores of dysmenorrhea, dyspareunia, and chronic pelvic pain were all significantly higher in PNI $(+)$ patients than in PNI $(-)$ patients $(P=0.031$, $P=0.024$, and $P=0.044$, respectively; Table $\mathrm{S} 2$ and Figure $1 \mathrm{C}$ ).

\section{Relationship between $\mathrm{PNI}$ and newly formed NFD in DIE}

Newly formed nerve fibers were positively stained by GAP43 through immunohistochemistry. NFD of newly formed nerve fibers was markedly increased in PNI $(+)$ group of patients with USL-DIE ( $P=0.001)$, compared with PNI (-) group (Table $\mathrm{S} 3$ and Figure $2 \mathrm{~A}$ and $\mathrm{B}$ ). A similar result was found in patients with RVS-DIE $(P=0.007)$ as well (Table S3 and Figure 2A and $\mathrm{C}$ ).
A

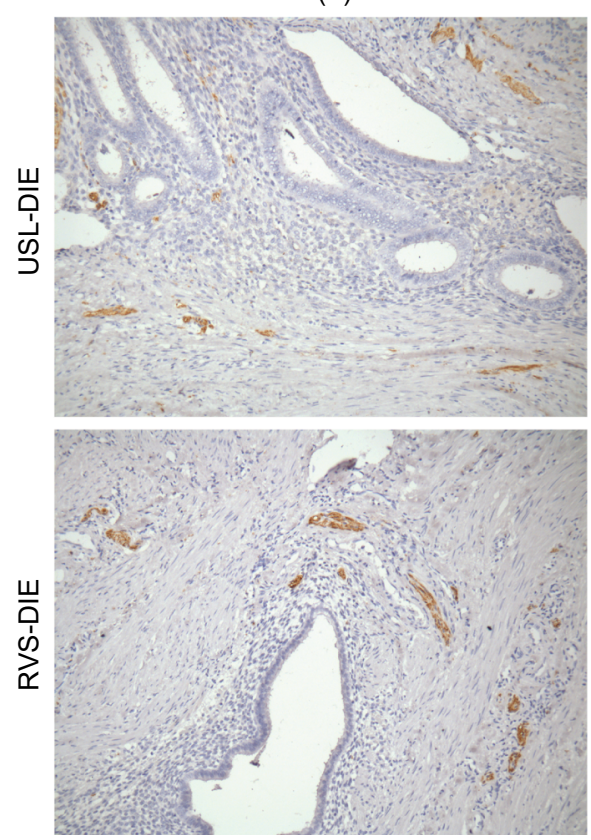

PNI (-)

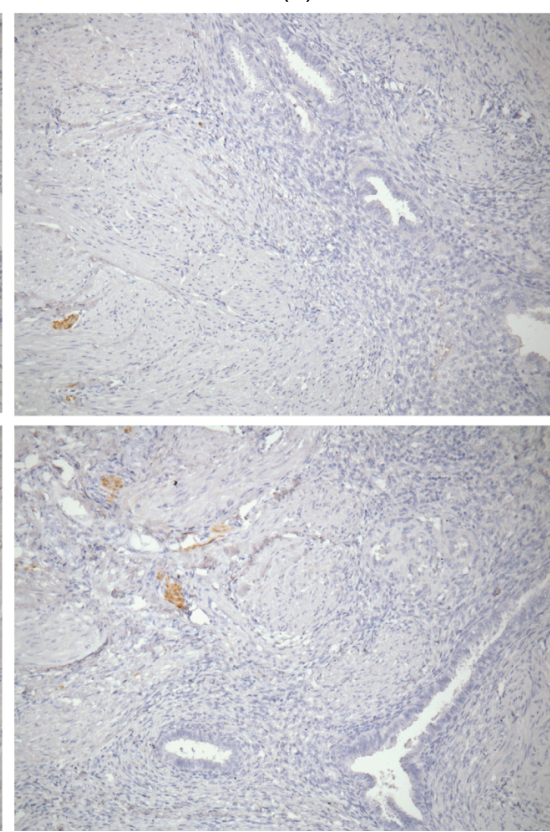

B

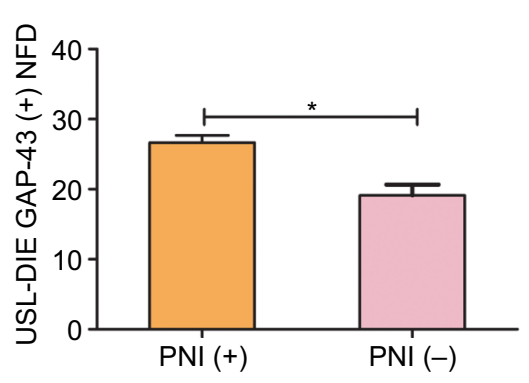

C

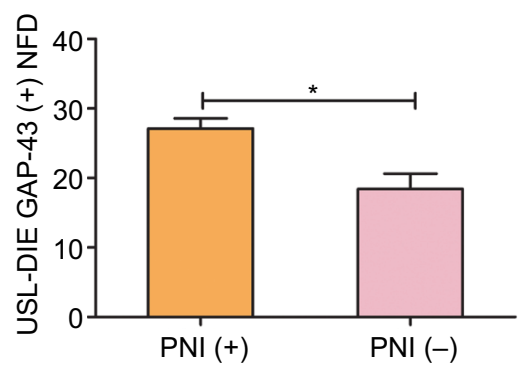

Figure 2 Detection of newly formed nerve fibers in lesions of USL-DIE and RVS-DIE.

Notes: Anti-GAP-43 was used to identify newly formed nerve fbers through immunohistochemistry (200x magnifcation). Newly formed nerve fibers were positively stained in USL-DIE and RVS-DIE lesions (A). NFDs of GAP-43 positively stained nerve fibers (shown in brown color) were significantly increased in lesions of PNI ( + ) group - (B) USL-DIE, $P=0.00 I$; (C) RVS-DIE, $P=0.007$ - compared with PNI (-) group. $* P<0.05$.

Abbreviations: USL-DIE, endometriosis infiltrating the uterosacral ligament; RVS-DIE, endometriosis involving the rectovaginal septum; Anti-GAP-43, antibody against growth-associated protein 43; NFD, nerve fiber density; PNI, perineural invasion. 


\section{Neuroangiogenesis accompanied by PNI in DIE}

More endothelial cells (CD31 positively stained, red) of microvessels were identified close to the nerve bundle (PGP 9.5 positively stained, green) within the endometriotic lesion (Figure 3A) of PNI (+) group. However, in endometriotic lesion without PNI, less endothelial cells were found (Figure 3B). Precise calculation and comparison of MVD between different groups further support the increased angiogenesis in PNI (+) lesions. MVDs in lesions of PNI (+) group of patients with USL-DIE and RVS-DIE were significantly higher than that of PNI (-) group ( $P=0.016$ and $P=0.001$, respectively; Table $\mathrm{S} 4$ and Figure $3 \mathrm{C}$ and $\mathrm{D})$.

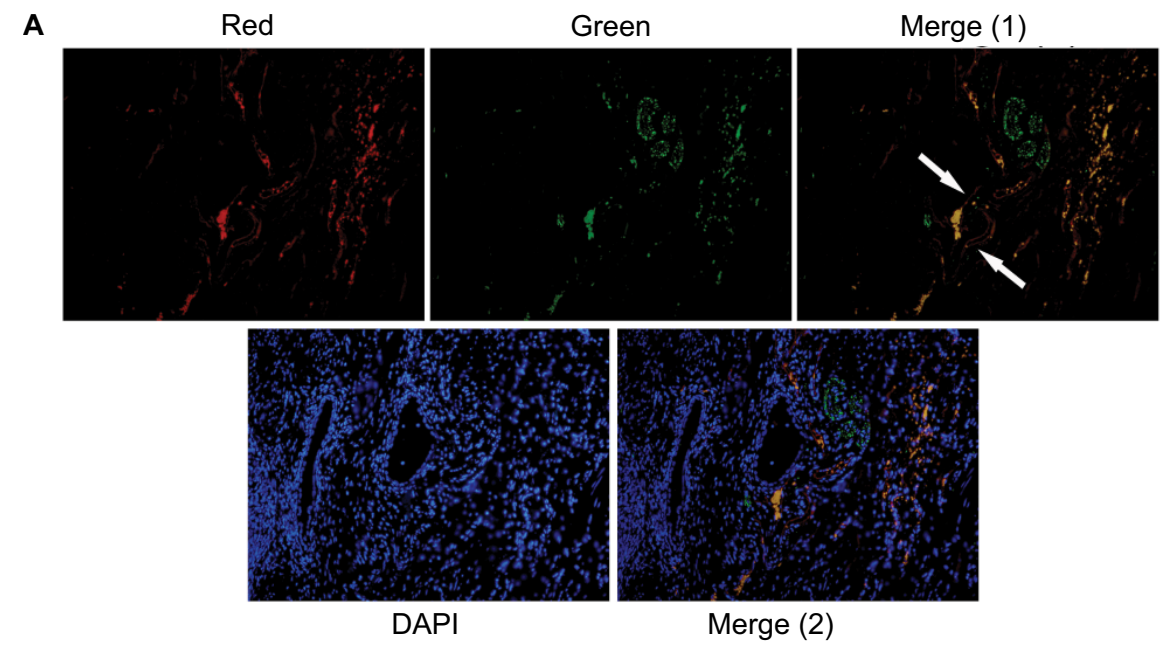

B

Red

Green

Merge (1)
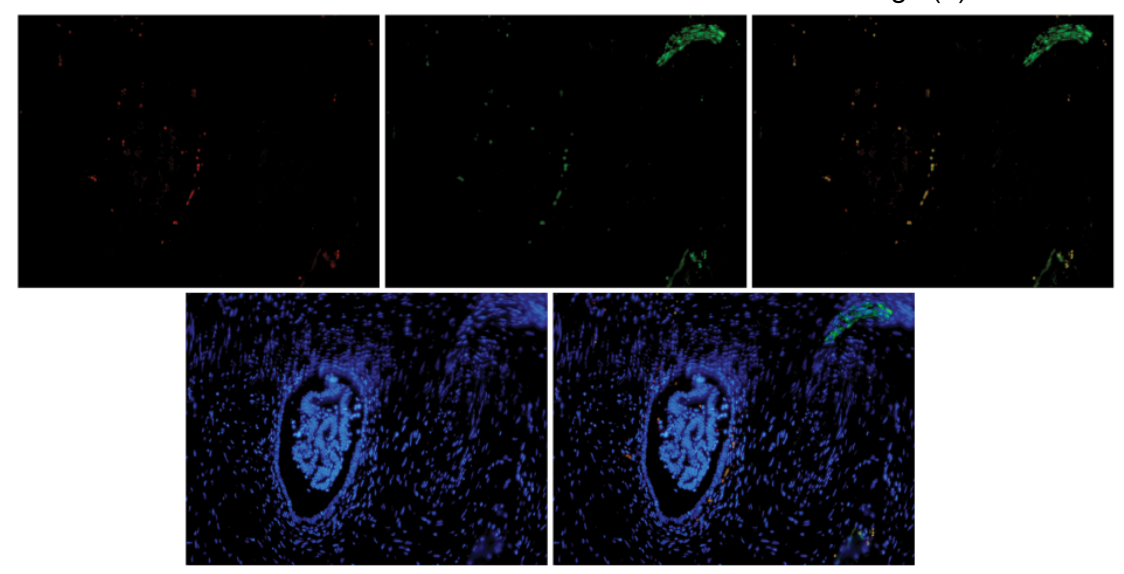

C

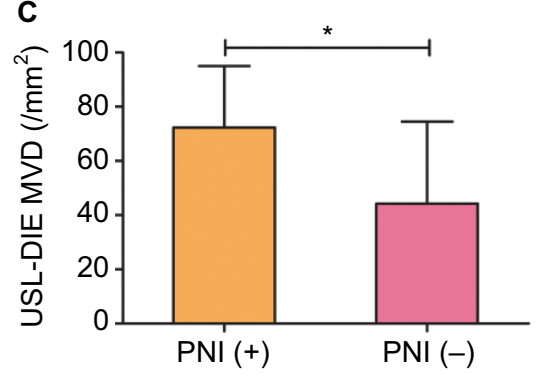

Merge (2)

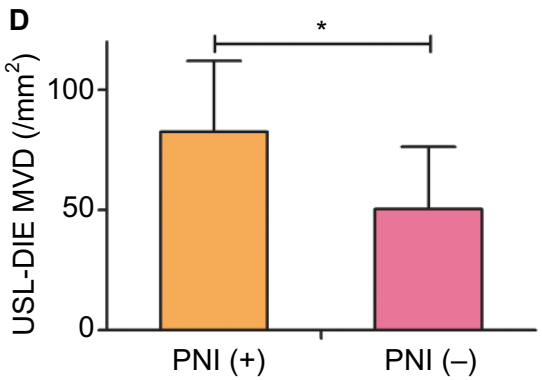

Figure 3 Distribution of microvessels in endometriotic lesions with/without PNI.

Notes: Double immunofluorescence was performed to present microvessels and nerve fibers simultaneously. Microvessels were identified by antibody against CD3I (a specific marker of endothelial cells), which was red-stained, whereas nerve fibers were identified by antibody against PGP 9.5 , which was green-stained. Nucleus was bluestained with DAPI. More microvessels were found in endometriotic lesions of the PNI (+) group (A), compared to that of the PNI (-) group (B). Exact quantization of the MVD was acquired in both USL-DIE and RVS-DIE lesions. Further comparison analysis showed similar results: there was an increased MVD in endometriotic lesions of the $\mathrm{PNI}(+)$ group than that of the PNI (-) group (C and D).

Abbreviations: PNI, perineural invasion; MVD, microvessel density; USL-DIE, endometriosis infiltrating the uterosacral ligament; RVS-DIE, endometriosis involving the rectovaginal septum. 
A

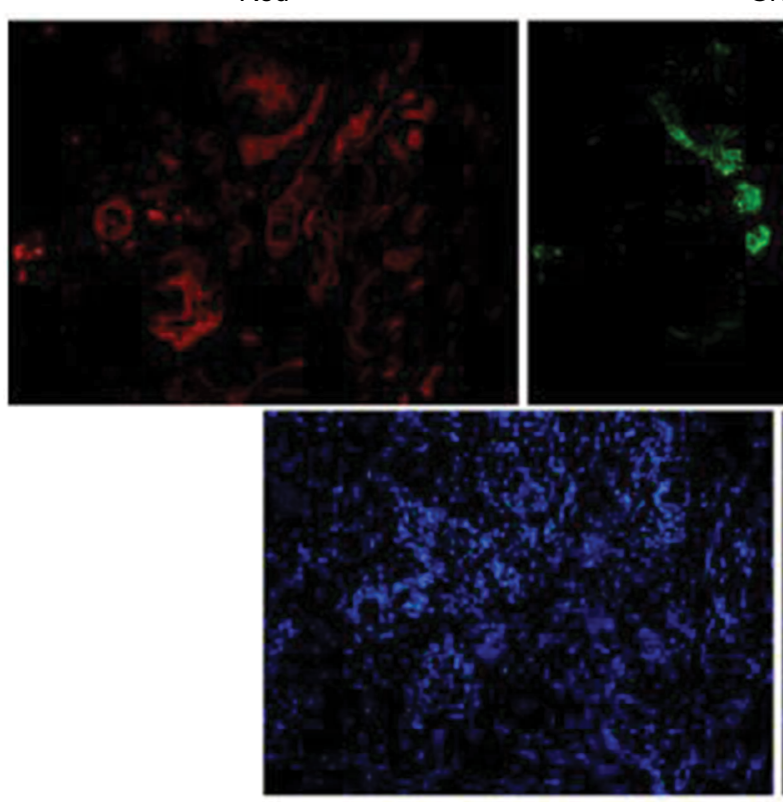

Green

Merge (1)
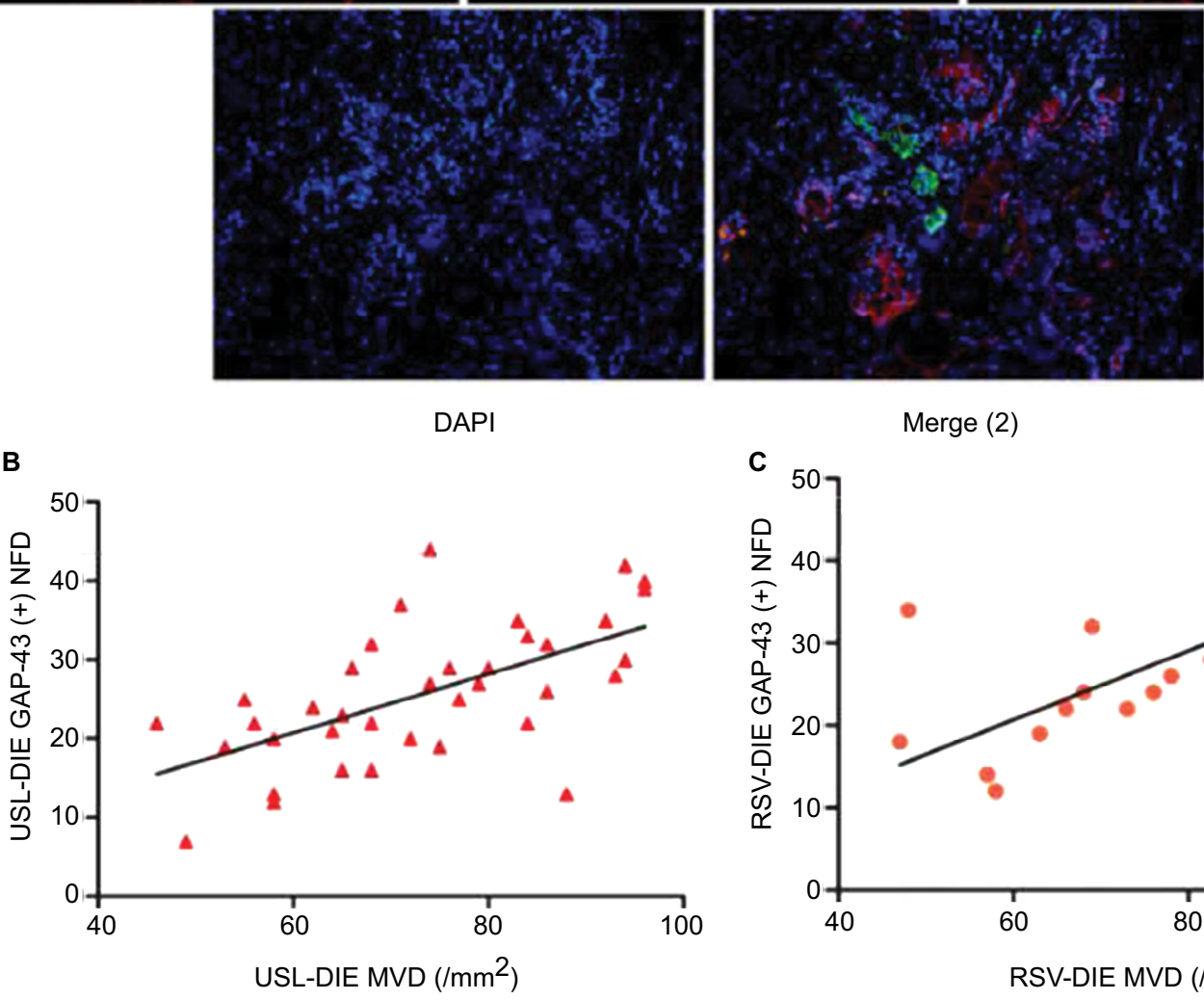

Merge (2)

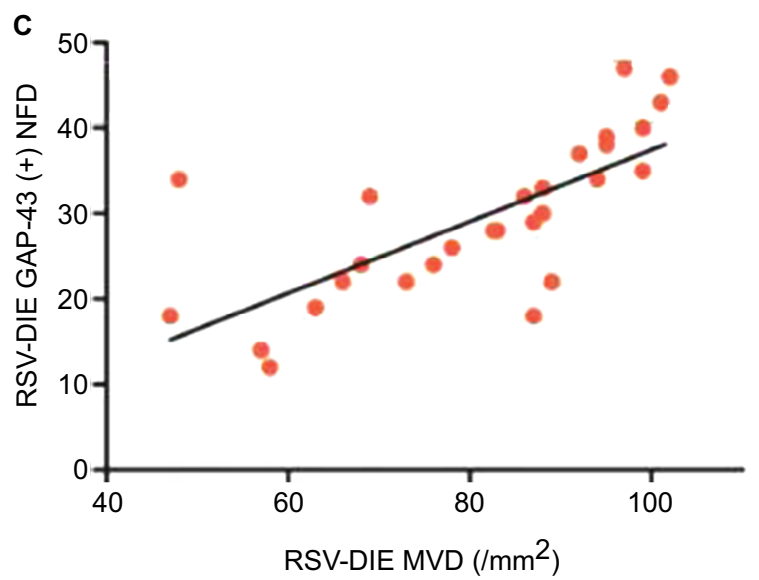

Figure 4 Relationship of neuroangiogenesis with PNI

Notes: (A) Double immunofluorescence was performed to present microvessel and newly formed nerve fibers simultaneously. Microvessels were red-stained (CD3। marked) and newly formed nerve fibers were green-stained (GAP-43 marked). The merged images showed a close nerve-vessel network in the endometriotic lesion. Further correlation analysis revealed that MVD in endometriotic lesions of the PNI (+) group was positively related to the NFD of newly formed nerve fibers; (B) USL-DIE, $P<0.00$ I; (C) RVS-DIE, $P<0.001$

Abbreviations: PNI, perineural invasion; MVD, microvessel density; NFD, nerve fiber density; USL-DIE, endometriosis infiltrating the uterosacral ligament; RVS-DIE, endometriosis involving the rectovaginal septum.

Double immunofluorescence was also performed to further elucidate the relationship between the newly formed nerve fibers and microvessels. Nerve fibers were formed along the microvessels, presenting a nerve-vessel network in the endometriotic lesion (Figure 4A). Moreover, NFD of newly formed nerve fibers in the lesion was positively related to the MVDs in the PNI (+) group of patients with USL-DIE and RVS-DIE, respectively $\left(R^{2}=0.382, P<0.001\right.$ and $R^{2}=0.524, P<0.001$, respectively; Figure $4 \mathrm{~B}$ and $\mathrm{C}$ ).

\section{Discussion}

DIE is defined as endometriotic lesions penetrating into the retroperitoneal space or the wall of the pelvic organs for a distance of $5 \mathrm{~mm}$ or more. ${ }^{18}$ Severe dysmenorrhea, dyspareunia, and chronic pelvic pain are most commonly found in patients with DIE nodules. The mechanisms underlying EAP are poorly understood. Some researchers believe that endometriosis-induced chronic inflammatory microenvironment may be the major reason initiating this pain-generating 
progress. ${ }^{19}$ However, more and more evidence supports that the generation of pain symptoms in endometriosis is multifactorial. Mechanisms involving the peripheral nervous system and the CNS seem to play a more important role in EAP.

Gynecologists have found that endometriotic lesion can develop its own sensory and sympathetic innervations. ${ }^{5,6}$ Arnold et al demonstrated a higher density of PGP 9.5 positively stained nerve fibers in peritoneal endometriotic lesions than in the peritoneum near the lesions. ${ }^{3}$ Wang et al showed that deep infiltrating endometriotic lesions were richly innervated by different nerve fibers (including sensory $\mathrm{A} \delta$, sensory $\mathrm{C}$, cholinergic and adrenergic nerve fibers). ${ }^{20}$ More importantly, they found a higher density of myelinated nerve fibers stained with NF in the deep infiltrating lesions than in peritoneal endometriotic lesions. Patients with pain symptoms had higher density of PGP $9.5(+)$ nerve fibers in peritoneal endometriotic lesions than those without pain, supporting a direct relationship between NFD and EAP. ${ }^{21}$ Mechsner et al found that patients with dysmenorrhea and/ or pelvic pain had a significantly higher frequency of nerve fibers in peritoneal endometriotic lesions than had patients without those symptoms. ${ }^{11}$ Our previous study further confirmed that the density of PGP $9.5(+)$ total nerve fibers is positively related with pain VAS scores both in patients with peritoneal endometriosis and DIE. ${ }^{12}$ All these data suggest that abnormal innervation in endometriosis is closely related with EAP.

Trauma to neural tissue produces abnormalities of neural function that are perceived by the patient as the symptoms and signs of pain. Accordingly, direct invasion or irritation of peripheral nerves by ectopic endometrial cells also contribute to the generation of pain in patients with endometriosis. Anaf et al demonstrated that there was a significantly higher proportion of perineural and intraneural invasion by endometriotic cells in patients with higher preoperative pain VAS scores than those with lower VAS scores. ${ }^{22}$ Their further study elucidated that higher expression of NGF in deep endometriotic nodules correlated with the proclivity of ectopic implants to invade the pelvic nerves. ${ }^{23} \mathrm{NGF}$ serves as one of the most important factors mediating the development, survival, and maintenance of sensory nerve fibers. Similar correlations were also found between TGF $\beta 1$ and the invasion of nerve fiber bundles in DIE lesions. ${ }^{24}$ TGF $\beta 1$ plays a key role in the immune response, angiogenesis, and endometrial stromal cell proliferation in endometriosis. ${ }^{25}$ The common characteristic of NGF and TGF $\beta 1$ is that they are secreted by inflammatory cells and highly expressed in ectopic endometrial cells. More importantly, they not only initiate and promote the inflamma- tory process of endometriosis but also induce local algesic stimulation in nerve fibers. The production of NGF can be increased by inflammatory mediators such as TNF- $\alpha$, IL-1, or IL-6. In turn, NGF can induce the degranulation of mast cells, releasing additional inflammatory factors such as serotonin and prostaglandin which sensitize peripheral nociceptors. ${ }^{26,27}$ TGF $\beta 1$ can induce endometrial cell invasion ${ }^{28}$ and facilitate the release of algesic substance from immune cells in the lesion. The increased density of immune cells near the nerve fibers and within lesion, and the more frequent presence of PNI in DIE lesions, suggest a potentially tri-directional role of the immune system (inflammatory effect), ectopic endometriotic cells, and nerve fibers in endometriosis-related pain. Other factors upregulated in endometriosis, including prostaglandin $\mathrm{F}^{29}$ bradykinin, ${ }^{30} \mathrm{NT}-3,{ }^{31}$ and $\mathrm{BDNF}^{32}$, may also trigger the illness inflammatory response and increase the sensitivity to noxious stimuli. It seems that nerve compression and distortion or damage by endometrial cells may be a crucial mechanism of inflammation around the lesion. ${ }^{33,34}$ However, inflammatory factors can inversely facilitate the invasive behavior of endometrial cells to endometriosisassociated nerve fibers. The invasion of the ectopic implant triggers the formation of a complicated interaction net. Eventually, a positive feedback loop is created and keeps going on (Figure 5). However, the reason why there exists a specific morphological relationship between nerves and endometriotic foci has not been satisfactorily elucidated.

This study also focused on investigating PNI in patients with DIE involving uterosacral ligament as well as rectovaginal septum. We identified that PNI was positive in $65.5 \%$ of USL-DIE patients and $70.0 \%$ of RVS-DIE patients. Moreover, PNI (+) patients had significantly higher pain VAS scores, indicating a direct relationship between PNI and EAP. Further analysis showed that PNI $(+)$ endometriotic foci have more abundant newly generated nerve fibers as well as microvessels than PNI (-) lesions. It was interesting to note that microvessels in PNI (+) lesions of DIE patients increased in parallel with the newly formed nerve fibers.

Investigators have shown that ectopic endometriotic lesions developed their own sensory and sympathetic innervations both in rats and women. ${ }^{5}$ Sympathetic as well as some classical sensory nerves play an important role in the generation of hyperalgesia. ${ }^{35}$ Yen et al found that primary afferent neurons in the periphery and dorsal root ganglia are abundantly surrounded by newly formed sympathetic sprouting due to neural invasion by ectopic implants. ${ }^{36} \mathrm{~A}$ chemical interaction between sympathetic and afferent 


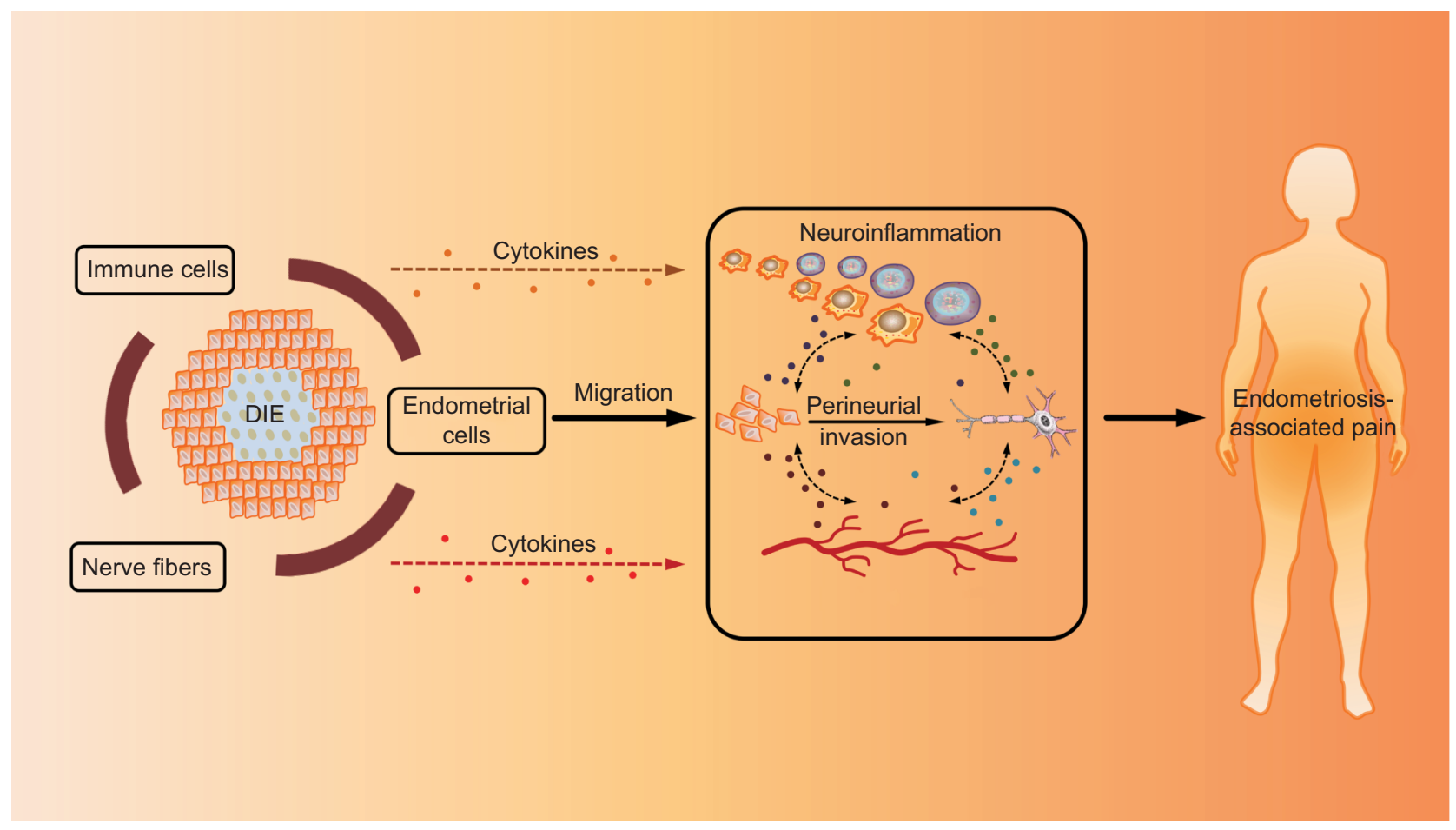

Figure 5 Mechanism of PNI in EAP.

Notes: The interaction between ectopic endometrial cells (including epithelial cells and stromal cells) and nerve fibers is believed to contribute to the sensitization of peripheral nerve fibers through multiple mechanisms, resulting in the formation of EAP. Once ectopic endometrial cells infiltrate nerve fiber in the lesion (PNI), neoangiogenesis and neurogenesis are simultaneously activated around the nerve fiber. This newly formed vasculature and innervation due to PNI in endometriotic lesions form a complex interaction network in the peritoneal microenvironment of endometriosis, which is called "neuroangiogenesis". In addition, the local immune system (containing immune cells, chemokines, cytokines, inflammatory factors, etc.) is also affected, leading to the dysfunction of immune cells as well as the synthesis and release of specific factors. On the one hand, the dysregulation of local immune system promotes the invasion activity of ectopic endometrial cells to nerve fibers. On the other hand, it can also increase factors mediating the development, survival, and maintenance of nerve fibers. More importantly, it forms an inflammatory environment that can sensitize peripheral nociceptors, which is called "neuroinflammation". Therefore, we propose a potentially tri-directional role of the immune system, ectopic endometriotic cells, and nerve fibers in EAP.

Abbreviations: $\mathrm{PNI}$, perineural invasion; EAP, endometriosis-associated pain; DIE, deep infiltrating endometriosis.

neurons is believed to be able to activate and/or sensitize nociceptors on the spinal cord dorsal horn neurons. As a consequence, noxious stimuli from endometriotic lesions are transformed to persistent nociceptive input and subsequently processed and postulated to the CNS, leading to persistent pain and hyperalgesia. ${ }^{37}$ Angiogenesis represents a critical step in the establishment and pathogenesis of endometriosis. Importantly, blood vessels are innervated by sensory and sympathetic fibers. ${ }^{38}$ Asante and Taylor proposed a new conception integrating "angiogenesis" and "neurogenesis", named "neuroangiogenesis", 8 in 2010, which they thought contributed to the survival and growth of ectopic implants, or even the generation of pain. The newly formed vasculature and innervation in endometriotic lesions form a complex interaction network in the peritoneal microenvironment of endometriosis. Several factors that can act on both sprouting blood vessels and nerve fibers (such as VEGF and VEGFR, semaphorin $3 \mathrm{~A}$ and its receptors, netrins and their DCC/ neogenin and Unc5 receptors, slits ligands and their roundabout [Robo] receptors, and ephrins and their Eph receptors) may play a potential role in this process. ${ }^{12,39-41}$ In addition, permeability of these newly formed immature vessels is increased by efferent actions from the CNS, leading to the process of neurogenic inflammation. As a result, activated macrophages and degranulating mast cells are abundantly recruited in endometriotic lesions. They can secrete neuroattractant cytokines, providing a suitable environment for the nerves to grow. However, future studies are needed to further investigate the role of these factors in the pathogenesis of endometriosis, and more importantly, the generation of pain.

Sensitized peripheral nerve fibers (nociceptors) are triggered in the inflammatory microenvironment of endometriosis and continuous injury input is transported to the CNS, initiating a process called central sensitization. ${ }^{42}$ However, due to the long-term "modification" of CNS functioning, central sensitization can become independent of peripheral sensitization via neural mechanisms similar to those underlying memory. ${ }^{43}$ That is why the patient may still feel pain long after the ectopic growths are removed. Additionally, the CNS can retain a "memory" of central neuronal changes induced by 
the endometriotic neural input. Local neural invasion-induced perception of pain may propagate to nociceptors near the lesion or even long away from the lesion. Due to the enormous CNS interconnectivity, "fake stimuli" still continue even after elimination of initiating pathophysiology and remind the memory of pain perception the patient suffered before.

\section{Conclusion}

PNI is commonly seen in many malignant diseases and some benign diseases. However, the observation of PNI is still poorly known in endometriosis. The pathophysiology of neural invasion by endometrial cells also needs to be further elucidated. The present study suggests that PNI is an important and frequent phenomenon in DIE. Moreover, PNI is closely related with EAP, including dysmenorrhea, dyspareunia, and chronic pelvic pain. Increased newly formed GAP-43 positive nerve fibers and microvessels play a crucial role in this morphological relationship, indicating a "neuroangiogenesis" mechanism underlying the generation of pain. Many factors can contribute interactively to individual variability in EAP. Nerve-immune cell interaction, nerve-endometrial cell interaction, and immune cell-endometrial cell interaction are all involved in the process of peripheral as well as central sensitization once PNI by ectopic implants occurs. Future investigations to improve the understanding on how these interactions influence pain experience in women with endometriosis are badly needed.

\section{Abbreviation list}

BDNF, brain-derived neurotrophic factor

$\mathrm{CNS}$, central nervous system

DIE, deep infiltrating endometriosis

EAP, endometriosis-associated pain

GAP-43, growth-associated protein 43

IL-1, interleukin-1

MVD, microvessel density

NFD, nerve fiber density

NGF, nerve growth factor

NT-3, neurotrophin-3

PGP 9.5, protein gene product 9.5

PNI, perineural invasion

RVS-DIE, endometriosis involving the rectovaginal septum

TGF $\beta 1$, transforming growth factor beta 1

TNF- $\alpha$, tumor necrosis factor- $\alpha$

USL-DIE, endometriosis infiltrating the uterosacral ligament

VAS, visual analog scale

VEGF, vascular endothelial growth factor

VEGFR, vascular endothelial growth factor receptor

\section{Acknowledgments}

The authors would like to thank Ms Ren Bingyan in assisting the paperwork of this manuscript. This work was supported by: 1) Natural Science Foundation of Guangdong Province, China (grant no. 2016A030310151); 2) Student Innovation Training Program of Sun Yat-sen University (grant no. 201701109); 3) Special Funds for the Cultivation of Guangdong College Students' Scientific and Technological Innovation (“Climbing Program" Special Funds, pdjh2017b0015); and 4) National Natural Science Foundation of China (Youth Science fund) (grant no. 81701416).

\section{Author contributions}

YL and SY designed the study and wrote the manuscript. DL, FY, WP, and FZ performed experiments and analyzed the data. JW, HX, and JL assisted with experimental techniques, analyzed the data, and polished the manuscript. All authors contributed toward data analysis, drafting and revising the paper and agree to be accountable for all aspects of the work.

\section{Disclosure}

The authors report no conflicts of interest in this work.

\section{References}

1. Stratton P, Berkley KJ. Chronic pelvic pain and endometriosis: translational evidence of the relationship and implications. Hum Reprod Update. 2011;17(3):327-346.

2. Arnold J, Barcena de Arellano ML, Rüster C, et al. Imbalance between sympathetic and sensory innervation in peritoneal endometriosis. Brain Behav Immun. 2012;26(1):132-141.

3. Arnold J, Vercellino GF, Chiantera V, Schneider A, Mechsner S, Barcena de Arellano ML. Neuroimmunomodulatory alterations in non-lesional peritoneum close to peritoneal endometriosis. Neuroimmunomodulation. 2013;20(1):9-18.

4. Tokushige N, Markham R, Russell P, Fraser IS. Nerve fibres in peritoneal endometriosis. Hum Reprod. 2006;21(11):3001-3007.

5. Berkley KJ, Dmitrieva N, Curtis KS, Papka RE. Innervation of ectopic endometrium in a rat model of endometriosis. Proc Natl Acad Sci USA. 2004;101(30):11094-11098.

6. Berkley KJ, Rapkin AJ, Papka RE. The pains of endometriosis. Science. 2005;308(5728):1587-1589.

7. Mechsner S, Schwarz J, Thode J, Loddenkemper C, Salomon DS, Ebert AD. Growth-associated protein 43-positive sensory nerve fibers accompanied by immature vessels are located in or near peritoneal endometriotic lesions. Fertil Steril. 2007;88(3):581-587.

8. Asante A, Taylor RN. Endometriosis: the role of neuroangiogenesis. Annu Rev Physiol. 2011;73:163-182.

9. Kajitani T, Maruyama T, Asada H, et al. Possible involvement of nerve growth factor in dysmenorrhea and dyspareunia associated with endometriosis. Endocr J. 2013;60(10):1155-1164.

10. Kobayashi H, Yamada Y, Morioka S, Niiro E, Shigemitsu A, Ito F. Mechanism of pain generation for endometriosis-associated pelvic pain. Arch Gynecol Obstet. 2014;289(1):13-21.

11. Mechsner S, Kaiser A, Kopf A, Gericke C, Ebert A, Bartley J. A pilot study to evaluate the clinical relevance of endometriosis-associated nerve fibers in peritoneal endometriotic lesions. Fertil Steril. 2009;92(6):1856-1861. 
12. Liang Y, Wang W, Huang J, et al. Potential Role of Semaphorin $3 \mathrm{~A}$ and Its Receptors in Regulating Aberrant Sympathetic Innervation in Peritoneal and Deep Infiltrating Endometriosis. PLoS One. 2015;10(12):e0146027.

13. Bourdel N, Alves J, Pickering G, Ramilo I, Roman H, Canis M. Systematic review of endometriosis pain assessment: how to choose a scale? Hum Reprod Update. 2015;21(1):136-152.

14. Merrilees AD, Bethwaite PB, Russell GL, Robinson RG, Delahunt B. Parameters of perineural invasion in radical prostatectomy specimens lack prognostic significance. Mod Pathol. 2008;21(9):1095-1100.

15. Potter SR, Partin AW. The significance of perineural invasion found on needle biopsy of the prostate: implications for definitive therapy. Rev Urol. 2000;2(2):87-90.

16. Jondet M, Vacher-Lavenu MC, Chapron C. Image analysis measurements of the microvascularisation in endometrium, superficial and deep endometriotic tissues. Angiogenesis. 2006;9(4):177-182.

17. Machado DE, Abrao MS, Berardo PT, Takiya CM, Nasciutti LE. Vascular density and distribution of vascular endothelial growth factor (VEGF) and its receptor VEGFR-2 (Flk-1) are significantly higher in patients with deeply infiltrating endometriosis affecting the rectum. Fertil Steril. 2008;90(1):148-155.

18. Koninckx PR, Meuleman C, Demeyere S, Lesaffre E, Cornillie FJ. Suggestive evidence that pelvic endometriosis is a progressive disease, whereas deeply infiltrating endometriosis is associated with pelvic pain. Fertil Steril. 1991;55(4):759-765.

19. Vercellini P, Somigliana E, Viganò P, Abbiati A, Daguati R, Crosignani PG. Endometriosis: current and future medical therapies. Best Pract Res Clin Obstet Gynaecol. 2008;22(2):275-306.

20. Wang G, Tokushige N, Markham R, Fraser IS. Rich innervation of deep infiltrating endometriosis. Hum Reprod. 2009;24(4):827-834.

21. Mckinnon B, Bersinger NA, Wotzkow C, Mueller MD. Endometriosisassociated nerve fibers, peritoneal fluid cytokine concentrations, and pain in endometriotic lesions from different locations. Fertil Steril. 2012;97(2):373-380.

22. AnafV, Simon P, El Nakadi I, et al. Relationship between endometriotic foci and nerves in rectovaginal endometriotic nodules. Hum Reprod. 2000;15(8):1744-1750.

23. Anaf V, Simon P, El Nakadi I, et al. Hyperalgesia, nerve infiltration and nerve growth factor expression in deep adenomyotic nodules, peritoneal and ovarian endometriosis. Hum Reprod. 2002;17(7):1895-1900.

24. Tamburro S, Canis M, Albuisson E, Dechelotte P, Darcha C, Mage G. Expression of transforming growth factor beta1 in nerve fibers is related to dysmenorrhea and laparoscopic appearance of endometriotic implants. Fertil Steril. 2003;80(5):1131-1136.

25. Zhang F, Yang Y, Wang Y. Association between TGF- $\beta 1-509 \mathrm{C} / \mathrm{T}$ polymorphism and endometriosis: a systematic review and meta-analysis. Eur J Obstet Gynecol Reprod Biol. 2012;164(2):121-126.

26. Jankowski MP, Koerber HR. Translational pain research: from mouse to man. In: Kruger L, Light AR, editors. Neurotrophic Factors and Nociceptor Sensitization. Boca Raton, FL: CRC Press/Taylor \& Francis; 2010:283-298.
27. Skaper SD, Facci L. $\left[{ }^{3} \mathrm{H}\right]$ serotonin release assay using antigen-stimulated rat peritoneal mast cells. Methods Mol Biol. 2012;846:333-341.

28. Liu YG, Tekmal RR, Binkley PA, Nair HB, Schenken RS, Kirma NB. Induction of endometrial epithelial cell invasion and c-fms expression by transforming growth factor beta. Mol Hum Reprod. 2009;15(10):665-673.

29. Ahmad SF, Akoum A, Horne AW. Selective modulation of the prostaglandin F2 $\alpha$ pathway markedly impacts on endometriosis progression in a xenograft mouse model. Mol Hum Reprod. 2015;21(12):905-916.

30. Yoshino O, Yamada-Nomoto K, Kobayashi M, et al. Bradykinin system is involved in endometriosis-related pain through endothelin-1 production. Eur J Pain. 2018;22(3):501-510.

31. Barcena de Arellano ML, Arnold J, Lang H, et al. Evidence of neurotrophic events due to peritoneal endometriotic lesions. Cytokine. 2013;62(2):253-261.

32. Wessels JM, Kay VR, Leyland NA, Agarwal SK, Foster WG. Assessing brain-derived neurotrophic factor as a novel clinical marker of endometriosis. Fertil Steril. 2016;105(1):119-128.e1-e5.

33. Chen S, Xie W, Strong JA, Jiang J, Zhang JM. Sciatic endometriosis induces mechanical hypersensitivity, segmental nerve damage, and robust local inflammation in rats. Eur J Pain. 2016;20(7):1044-1057.

34. Bove GM. A model for radiating leg pain of endometriosis. $J$ Bodyw Mov Ther. 2016;20(4):931-936.

35. Zhang G, Dmitrieva N, Liu Y, McGinty KA, Berkley KJ. Endometriosis as a neurovascular condition: estrous variations in innervation, vascularization, and growth factor content of ectopic endometrial cysts in the rat. Am J Physiol Regul Integr Comp Physiol. 2008;294(1): R162-R171.

36. Yen LD, Bennett GJ, Ribeiro-da-Silva A. Sympathetic sprouting and changes in nociceptive sensory innervation in the glabrous skin of the rat hind paw following partial peripheral nerve injury. J Comp Neurol. 2006;495(6):679-690.

37. Bajaj P, Bajaj P, Madsen H, Arendt-Nielsen L. Endometriosis is associated with central sensitization: a psychophysical controlled study. J Pain. 2003;4(7):372-380.

38. Burnstock G. Autonomic neurotransmission: 60 years since sir Henry Dale. Anпu Rev Pharmacol Toxicol. 2009;49:1-30.

39. Carmeliet P, Tessier-Lavigne M. Common mechanisms of nerve and blood vessel wiring. Nature. 2005;436(7048):193-200.

40. Jones CA, Li DY. Common cues regulate neural and vascular patterning. Curr Opin Genet Dev. 2007;17(4):332-336.

41. Raab S, Plate KH. Different networks, common growth factors: shared growth factors and receptors of the vascular and the nervous system. Acta Neuropathol. 2007;113(6):607-626.

42. Woolf CJ, Salter MW. Neuronal plasticity: increasing the gain in pain. Science. 2000;288(5472):1765-1769.

43. Ren K, Dubner R. Pain facilitation and activity-dependent plasticity in pain modulatory circuitry: role of BDNF-TrkB signaling and NMDA receptors. Mol Neurobiol. 2007;35(3):224-235. 


\section{Supplementary materials}

Table SI Relationship between PNI and pain symptoms in patients with USL-DIE

\begin{tabular}{llll}
\hline Group & PNI (+) & PNI (-) & P-value \\
& $\mathbf{n = 3 8}$ & $\mathbf{n = 2 0}$ & \\
\hline Dysmenorrhea & $6.4 \pm 2.9$ & $4.4 \pm 3.1$ & $0.020^{*}$ \\
Dyspareunia & $3.5 \pm 2.8$ & $2.3 \pm 2.6$ & 0.054 \\
Chronic pelvic pain & $4.7 \pm 3.2$ & $2.7 \pm 2.4$ & $0.011^{*}$ \\
\hline
\end{tabular}

Note: $* P<0.05$.

Abbreviations: PNI, perineural invasion; USL-DIE, endometriosis infiltrating the uterosacral ligament.

Table S2 Relationship between PNI and pain symptoms in patients with RSV-DIE

\begin{tabular}{llll}
\hline Group & $\begin{array}{l}\text { PNI } \\
(+)\end{array}$ & $\begin{array}{l}\text { PNI (-) } \\
\mathbf{n = 1 2}\end{array}$ & P-value \\
& $\mathbf{n = 2 8}$ & & \\
\hline Dysmenorrhea & $6.6 \pm 3.0$ & $4.0 \pm 3.2$ & 0.03 I $^{*}$ \\
Dyspareunia & $5.1 \pm 2.5$ & $3.3 \pm 2.4$ & $0.024^{*}$ \\
Chronic pelvic pain & $5.8 \pm 2.4$ & $3.3 \pm 2.7$ & $0.044^{*}$ \\
\hline
\end{tabular}

Note: $* P<0.05$.

Abbreviations: PNI, perineural invasion; RVS-DIE, endometriosis involving the rectovaginal septum.
Table S3 Relationship between PNI and newly formed NFD in patients with USL-DIE and RSV-DIE

\begin{tabular}{llll}
\hline GAP-43 (+) & PNI $(+)$ & PNI (-) & P-value \\
NFD & & & \\
\hline USL-DIE & $26.66 \pm 6.42(\mathrm{n}=38)$ & $19.10 \pm 7.00(\mathrm{n}=20)$ & $0.00 I^{*}$ \\
RSV-DIE & $27.12 \pm 7.56(\mathrm{n}=28)$ & $18.42 \pm 7.61(\mathrm{n}=12)$ & $0.007^{*}$ \\
\hline
\end{tabular}

Note: $* P<0.05$.

Abbreviations: PNI, perineural invasion; NFD, nerve fiber density; USL-DIE, endometriosis infiltrating the uterosacral ligament; RVS-DIE, endometriosis involving the rectovaginal septum.

Table S4 Relationship between PNI and newly formed nerve fiber density in patients with USL-DIE and RSV-DIE

\begin{tabular}{llll}
\hline MVD & PNI (+) & PNI (-) & $P$-value \\
\hline USL-DIE & $72.4 \pm 22.6(n=38)$ & $44.3 \pm 30.2(n=20)$ & $0.016^{*}$ \\
RSV-DIE & $82.6 \pm 29.5(n=28)$ & $50.4 \pm 25.9(n=12)$ & $0.001^{*}$ \\
\hline
\end{tabular}

Note: $* P<0.05$.

Abbreviations: PNI, perineural invasion; USL-DIE, endometriosis infiltrating the uterosacral ligament; RVS-DIE, endometriosis involving the rectovaginal septum; MVD, microvessel density.
Journal of Pain Research

\section{Publish your work in this journal}

The Journal of Pain Research is an international, peer reviewed, open access, online journal that welcomes laboratory and clinical findings in the fields of pain research and the prevention and management of pain. Original research, reviews, symposium reports, hypothesis formation and commentaries are all considered for publication.

\section{Dovepress}

The manuscript management system is completely online and includes a very quick and fair peer-review system, which is all easy to use. Visit http://www.dovepress.com/testimonials.php to read real quotes from published authors. 